Original Research Paper

\title{
Automatic Piano Sheet Music Transcription with Machine Learning
}

\author{
${ }^{1}$ Fernandes Saputra, ${ }^{1}$ Un Greffin Namyu, ${ }^{1}$ Vincent, ${ }^{1}$ Derwin Suhartono and ${ }^{2}$ Aryo Pradipta Gema \\ ${ }^{1}$ Computer Science Department, School of Computer Science, Bina Nusantara University, Jakarta, 11480, Indonesia \\ ${ }^{2}$ Epigene Labs, 7 Square Gabriel Fauré, Paris 75017, France
}

\author{
Article history \\ Received: 23-09-2020 \\ Revised: 02-03-2021 \\ Accepted: 03-03-2021 \\ Corresponding Author: \\ Derwin Suhartono \\ Computer Science Department, \\ School of Computer Science, \\ Bina Nusantara University, \\ Jakarta, 11480, Indonesia \\ Email: dsuhartono@binus.edu
}

\begin{abstract}
Automatic Music Transcription (AMT) is becoming more and more popular throughout the day, it has piqued the interest of many in addition to academic research. A successful AMT system would be able to bridge multiple ranges of interactions between people and music, including music education. The goal of this research is to transcribe an audio input to music notation. Research methods were conducted by training multiple neural networks architectures in different kinds of cases. The evaluation used two approaches, those were objective evaluation and subjective evaluation. The result of this research was an achievement of $74.80 \% \mathrm{~F} 1$ score and $73.3 \%$ out of 30 respondents claimed that Bidirectional Long Short-Term Memory (BiLSTM) has the best result. It could be concluded that BiLSTM is the best architecture suited for automatic music transcription.
\end{abstract}

Keywords: Automatic Music Transcription, Recurrent Neural Network, Convolutional Neural Network

\section{Introduction}

Artificial intelligence has been a hot topic as of late due to its powerful capabilities in helping humans, as such, a lot of applications are now applying it as their base program. Multiple products of artificial intelligence could be seen in various fields, one of its best features is data mining, analyzing chunks of massive data then returning a particular characteristic from that data.

The popular feature of extracting characteristics is also widely used in other scopes of artificial intelligence. Currently, there has been a lot of research that also challenges artificial intelligence in the topic of voice processing. As the name suggests, voice processing is the ability of artificial intelligence to retrieve useful information or any characteristic that it could extract from the input, which here is a signal that was converted from the given audio (Joshi et al., 2017). The program is going to receive the audio which then analyzed and turned into a chain of signals so that the machine could understand and process. This field has a close relation with Automatic Music Transcription (AMT). AMT focuses on how to compute and convert a given audio input into a music signal which later transforms into music notation (sheet music). In respect to voice processing, AMT is called the musical equivalent of automatic voice recognition, in the sense that both tasks involve converting signals to symbolic sequences (Benetos et al., 2018). It has been one of the many challenging tasks even for an artificial intelligence model to discern the audio input regardless of the noises, frequency, etc. and finally return the expected music notation.

Furthermore, AMT is also closely associated with image processing, as sometimes, musical objects, usually music notation, could be recognized as a two-dimensional pattern with respect to time and frequency, widely known as a spectrogram. From the spectrogram that audio generated, it was then treated as the input of an image processing artificial intelligence where the characteristic of the spectrogram is going to be extracted and identified.

As AMT becomes more and more popular throughout the day, it has piqued the interest of many in addition to academic research. A successful AMT system would be able to bridge multiple ranges of interactions between people and music, including music education (by producing sheet music, it helps in the process of education), music creation, music production, music search, musicology (Benetos et al., 2018).

\section{Problem Definition}

The ability to transcribe music audio into music notation (sheet music) is one of many fascinating examples of human intelligence. It requires perception in 
analyzing complex auditory scenes, cognitive ability (recognizing musical objects), previous knowledge of the corresponding theory and interference when testing new combinations in music notation (Benetos et al., 2018). However, not many have that kind of capability, some people are still struggling to even define what note the audio is playing on. Furthermore, when listening to music by ear, it is quite hard to pin-point the exact and correct note. This is also the same as how different artificial intelligence's architectures or algorithms will have the result, not all artificial intelligence has the capabilities to process audio and have a great result at the end. Therefore, this research will address which of the available algorithms available will be able to give the best result to perform an audio processing task. The problems that could be derived from the statements above are: What is the difference in the F1-score used between Convolutional Neural Network (CNN), Long Short-Term Memory (LSTM), Multilayer Perceptron (MLP), Deep Neural Network (DNN) and Bidirectional Long Short-Term Memory (BiLSTM)? And subsequently what is the most suitable neural network architecture in processing sequential data (music) for automatic music transcription?

\section{Related Works}

In the first paper from (Sigtia et al., 2016), the paper proposed an architecture for an end-to-end approach of transcribing a piece of piano music into music notes. The proposed model is a combination of an acoustic model of CNN with the addition of music language model RNN NADE which is later compared with other architectures. This paper used full musical pieces from the MIDI Aligned Piano Sounds (MAPS) (Emiya et al., 2010) dataset which contained 270 pieces of classical music and MIDI annotations for training and testing the models in which 210 of the pieces are synthesized recordings and the rest are real recordings. The configuration for the training and testing was $80 \%$ of the data for training (216 musical pieces) and the remaining $20 \%$ is for testing (54 pieces). The paper transforms the input audio to a time-frequency representation where the representation chosen is Constant Q Transform (CQT) over Short Time Fourier Transform (STFT). There are 2 reasons for choosing CQT over STFT, the first reason is that CQT has much lower dimensional representation than the STFT in which it is useful when using neural network acoustic models as it reduces the number of parameters in the model and the second reason is that CQT is better suited as time-frequency representation for music signals, since the frequency axis is linear in pitch.

The next paper is by ( $\mathrm{Li}$ et al., 2017) which observed DNN and LSTM in music transcription tasks. The authors claimed that it is inefficient to deal with the audio files directly and therefore it will be transformed into spectrograms to extract features. The method used in this research is CQT due to its advantages as it provides a logarithmic frequency domain and is better in other reports.

This research also uses the MAPS dataset specifically the MUS part (pieces of music) as the authors claim it is an outstanding dataset due to each .wav file having a corresponding text document with ground truth for all pitches. The total number of pieces of music is 270 (around $11 \mathrm{~GB}$ ) and the data is split into training, validation and test sets with 60:20:20 percentage ratio. Using the MAPS dataset, music files are down sampled to $16 \mathrm{kHz}$ providing a 32-millisecond duration per frame. There are 252 features which account for 36 features per octaves. Labels are also generated with 88 labels for each frame. The DNN architecture uses a ReLU activation function for hidden layers and a sigmoid activation function for the output. LSTM uses hyperbolic tangent activation function hidden layers and sigmoid activation function for the output layer.

Another paper is from (Hawthorne et al., 2017), in this paper tried to make a transcription pipeline from an audio into a MIDI file. It uses two architectures to predict two different things which are combined on a certain stage to not only predict when notes are played but also the onset of those notes. The paper itself uses MAPS datasets which consists of music created with synthesizers and music created with digital piano and the authors of this paper also ensure that the same music piece was not present in more than one set.

The onset and frame detectors used in this study are built upon the convolutional layer and will be followed by a bidirectional LSTM with 128 units in both directions (forward and backward) and then connected to a fully connected sigmoid layer with 88 outputs to represent the 88 piano keys. The output of the onset detector is then concatenated with the output of a fully connected sigmoid of the frame activation detector which is then followed by BiLSTM. Finally, the output of it is followed by a fully connected sigmoid layer with 88 outputs.

The authors also compared the proposed architecture with other similar works and had the best result. Furthermore, the authors also discussed the need for more data and more rigorous evaluation. In this paper, the authors claimed that MAPS dataset has the main drawback of not providing the needed large dataset to best measure transcription quality and therefore will not be able to further improve the result. There are a lot of papers restricting the data used in evaluation by only using the first $30 \mathrm{sec}$ or less of each file. This is due to only using the "close" collection from each of the test sets and improving the evaluation score. However, this method was believed to result in an evaluation that is not representative to transcript the real-world transcription task. 
Kelz et al. (2016) attempted to explore the limitation of simple approaches in the task of automatic music transcription in which the authors conduct an in-depth analysis of neural network based framewise transcription with detailed analysis of the most suitable input representations for transcription systems in neural networks and other hyper-parameter tuning that would greatly affect the performance of the neural network. The dataset used in these experiments is the MAPS dataset. It provides MIDI-aligned recordings of a lot of classical music pieces. The pieces were rendered using high quality piano samples as well as real recording from Disklavier. The author claimed that it ensured clean annotation and therefore will have almost no labelnoise. The configuration dataset for training and testing is split to $80: 20 \mid$ percent of each of the categories. This research tested DNN, ConvNet and AllConv with different types of hyper parameter tuning for around 3000 runs with a Tesla K40 GPU and each run was roughly 8-9 h resulting in a total of 900 days of compute time for this research. The results of all the runs were that the most important hyper-parameters are learning rate and spectrogram type. This paper also used the practical recommendation for training the neural networks with Stochastic Gradient Descent (SGD) and the author claimed that strategies that dynamically adapt the learning rate such as Adam helped to a certain extent, but still not spare the tuning of the initial learning rate and its schedule.

A paper from (Dua et al., 2020), also uses RNNLSTM model to estimate sheet music generation. The highlighted method from this research is, to improve the accuracy of generated sheet music by using source separation and chord separation. Source separation separates the vocal and non-vocal part from the audio. Chord estimation can be done by dividing the non-vocal part into drums, bass, melodies and etc. This method is useful for amateurs who just started in learning music and relies heavily on sheet music such as songs that are not too popular such as songs from local bands may not have sheet music at all.

The DSD100 dataset has 100 songs with $44.1 \mathrm{kHz}$ sample rate each with the non-vocal part separated into its component which are bass, drum and other components. Features used to train the model are the magnitudes of the spectrogram of audio signals. Using magnitude spectra of the mixed signal and magnitude spectra of the target sources which are bass, drum, music and vocal as an input. The output of the network for each epoch are the estimated signal of each source (bass, drum, music and vocal).

Mixed signals are passed on to a Multi cell RNN containing 3 GRU cells with each of them having 256 units. This RNN model is connected to a dense layer with Rectified Linear Unit (ReLU) as the activation function that outputs the estimated signal of each source.

For chord estimation, pitch is the basic unit of a chord which is a function of the sound's fundamental frequency. A set of harmonic pitches that contains two or more pitches is called a chord containing the non-vocal part obtained from the source separation method makes a combination of pitches which means that several chords are also played in a series. The pitches are then passed on to a trained feature extractor model to estimate the chords.

\section{Dataset and Features}

\section{Dataset}

In this project, the data that are going to be used for training is in the form of music files. The music files or dataset are collected from MIDI Aligned Piano Music or widely known as MAPS dataset as the current best dataset that is available in the formats of music such as random chords, random notes and real pieces of music from famous composers such as Mozart, Chopin and Beethoven. The dataset also provides a different recording environment including large room, close and digitally recorded music. The dataset which is used will be the real pieces of music which include 270 files. Music files are in WAV format with its corresponding MIDI file and a text file that explains which pitches were pressed on the piano and also the onset and offset time. On this stage, music file names are looked up and are stored in an array. These files will then be opened on the next stage to be processed. The data coming from a music file can be represented with a one-dimensional array for mono and two-dimensional array for stereo (left and right channel) of floats with the length of seconds times sampling rate.

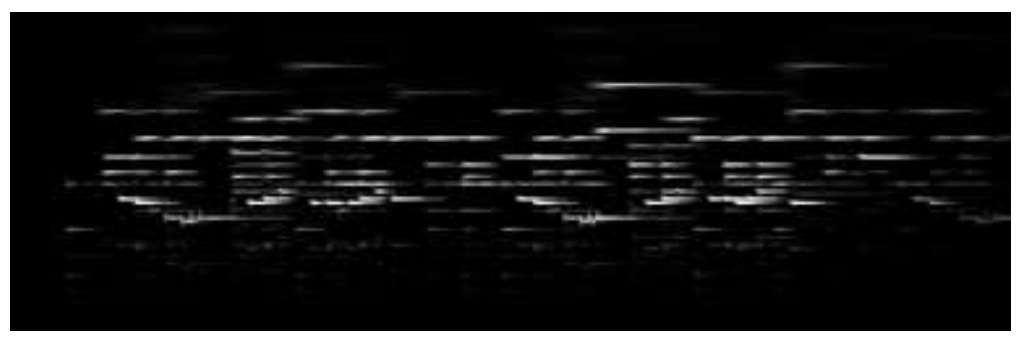

Fig. 1: CQT Representation from a music file 


\section{Features}

Figure 1 is an example of CQT that was generated from a music file. Constant-Q Transform (CQT) is closely related to the Fourier transform which has the same function of mapping frequencies. The difference is that CQT has geometrically spaced center frequencies. Which is very useful because every MIDI pitch also has geometrically spaced frequencies:

$f_{k}=f_{0} \cdot 2^{\frac{k}{b}}(k=0, \ldots)$

where, $b$ denotes the number of filters per octave. To make the filter domain adjacent to each other, the $k$-th filter is chosen as:

$$
\Delta_{k}^{c q}=f_{k+1}-f_{k}=f_{k}\left(2^{\frac{1}{b}}-1\right)
$$

This calculation resulting in a constant ratio of frequency to resolution:

$$
Q=\frac{f_{k}}{\Delta_{k}^{c q}}=\left(2^{\frac{1}{b}}-1\right)^{-1}
$$

The advantages of CQT compared to Fourier transform is that if the number of filters per octave is 12 and $f_{0}$ which is the minimal center frequency as the frequency of MIDI pitch of 0 , then the $k$-th $c q$-bin will correspond to the MIDI pitch of $k$ (Blankertz, 2001).

When this step is finished, we are left with a similar two-dimensional array shape across architectures, which shape is 252 features and some number of frames depending on time annotated by -1 .

\section{Labels}

To generate labels, a different approach is used by different code bases. For instance, LSTM and MLP the labels are generated by looking at provided text files such as Fig. 1 and for $\mathrm{CNN}$, the labels are generated by parsing the MIDI files.

In piano, the number of possible pitches is 88 . The numbers from the MidiPitch column represent a vector that has a dimension of 88 . With this, the label of each frame can be set to one if the MidiPitch is played in the current frame and zero if otherwise. These files are then saved with each file having frames corresponding to the audio. This means if the audio frames are set to 50000 frames then the label will also have 50000 frames. With this, each audio file has its corresponding label.

This step will produce a two-dimensional array like the generated CQT from the previous step. This matrix has the shape of 88 labels and some number of frames depending on time annotated by -1 .

\section{Methods}

\section{Multi-Layer Perceptron and Deep Neural Network}

In standardization, the mean and standard deviation are calculated for each feature and the values are then subtracted by mean and divided by the standard deviation resulting in data with a standard deviation of one. This is done to avoid common problems known as vanishing gradient and exploding gradient where the data are too small or too far apart.

The formula is shown below:

$$
x_{i_{-} \text {new }}=\frac{x_{i}-\bar{x}_{i}}{s t d_{i}}
$$

This is done without modifying the shape of the CQT. MLP and DNN are the same. The difference in name is made to differentiate the approach used to build the model. In MLP a naive approach is used which can be used as a basis. By naive, it means that there is no consideration to fight back overfitting. In Fig. 2, there is only one hidden layer with 100 nodes for each classifier or label and this approach is called a one-versus-all approach. A one-versus-all approach is basically training several binary classifier models simultaneously to each predict a certain pattern. As this problem has 88 expected labels, there are 88 binary classification models. Each having a hidden layer with 100 nodes with ReLU activation and 1 node output with sigmoid.

In the case of DNN as shown in the figure, there are 3 hidden dense layers used for training as shown in Fig. 3 with Deep Neural Network with each layer having 256 units paired with a dropout layer at the rate of 0.2. Each hidden layer is using ReLU activation function and the output was set using a sigmoid function. The loss function is Mean Squared Error.

\section{Convolutional Neural Network}

The model used for $\mathrm{CNN}$ is taken from previous research of (Bereket and Shi, 2017). As shown in Fig. 4, the input is in the form of $(252,7,1)$ which is the result of a windowed CQT. The input CQT is in the shape of $(252,7,50)$. The first convolutional layer has 50 filters with a kernel size of $(5,25)$ maintaining its original size and activation function of hyperbolic tangent followed by a max pooling layer sized $(1,3)$ reducing the size to $(84,7,50)$. The second convolutional layer also has 50 filters with the same max pooling layer size and dropout rate but with a different kernel size which is $(3,5)$ where 3 and 5 is along the frame axis and frequency axis outputting the same shape $(84,7,50)$ which then put through a max pooling with the shape of $(1,3)$ for the second time down sampling it to $(28,7,50)$. After that, it will be flattened resulting in the shape of (9800) and 
followed by two fully connected layers with each layer using sigmoid functions. The first fully connected layer or the third layer consists of 1000 hidden nodes and the second fully connected layer or the fourth layer, consists of 200 nodes. The output layer has 88 nodes with sigmoid function which is the same as the label generated so that the output can be compared to which
MIDI pitch is activated in the label. Binary cross entropy loss function is used for each node. The structures used are the exact same as the one used in (Bereket and Shi, 2017). As the dataset used in the paper is different, the models are run again with the same MAPS dataset and configurations (epoch and early stops) to unify the input and expected output in order to make it comparable.

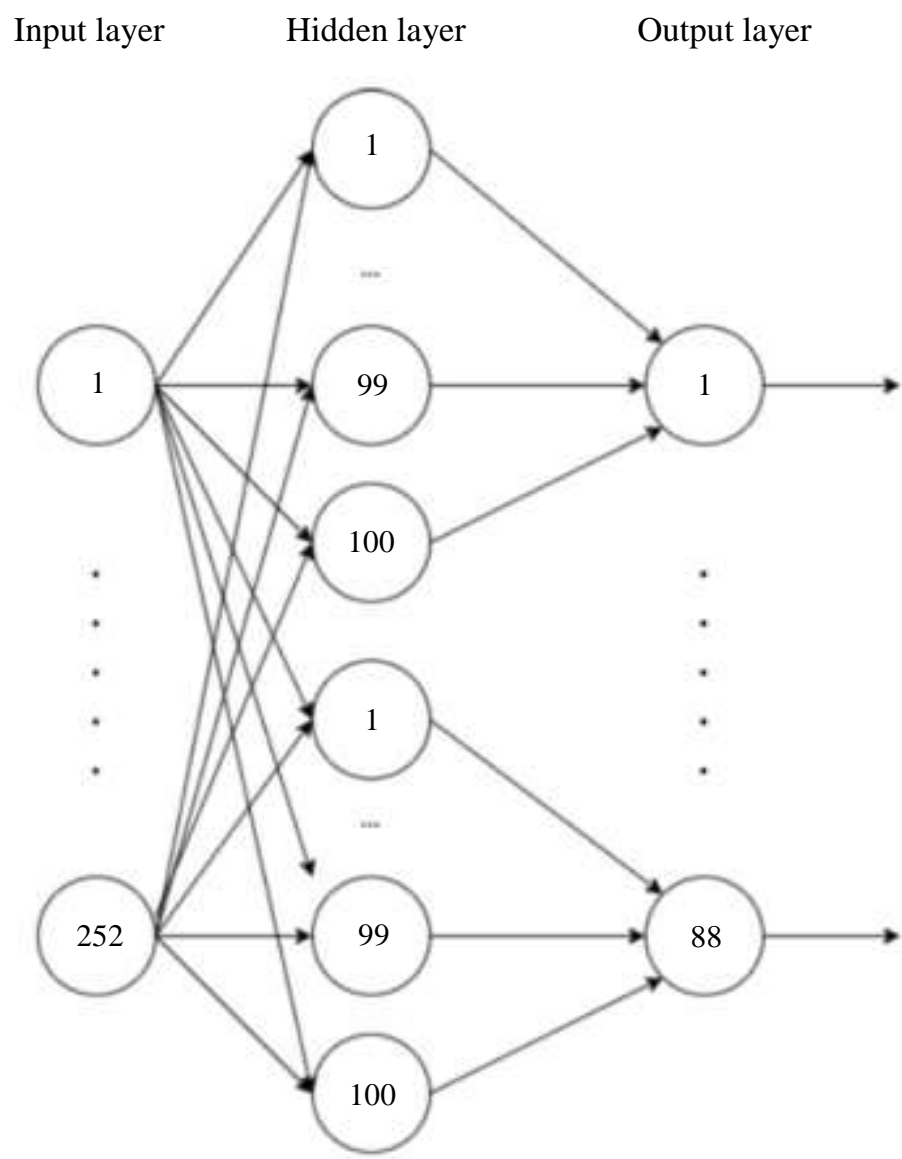

Fig. 2: MLP One-Versus-All Architecture

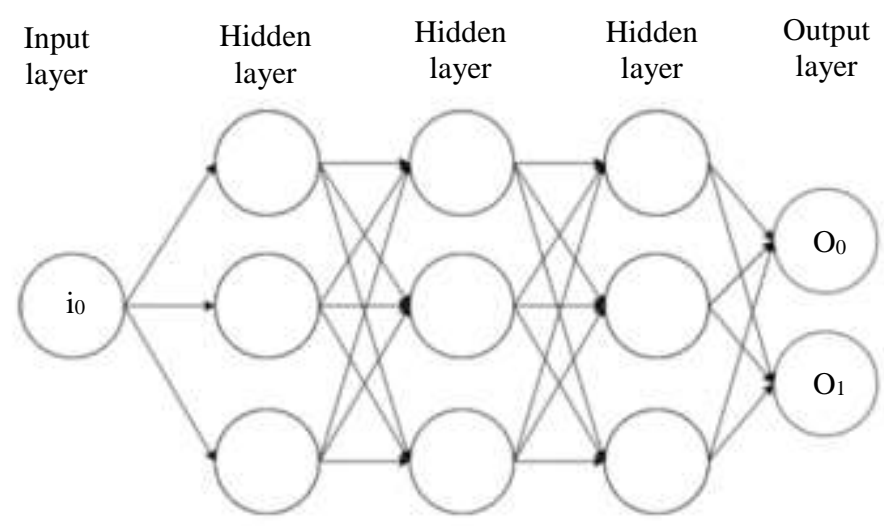

Fig. 3: DNN architecture 


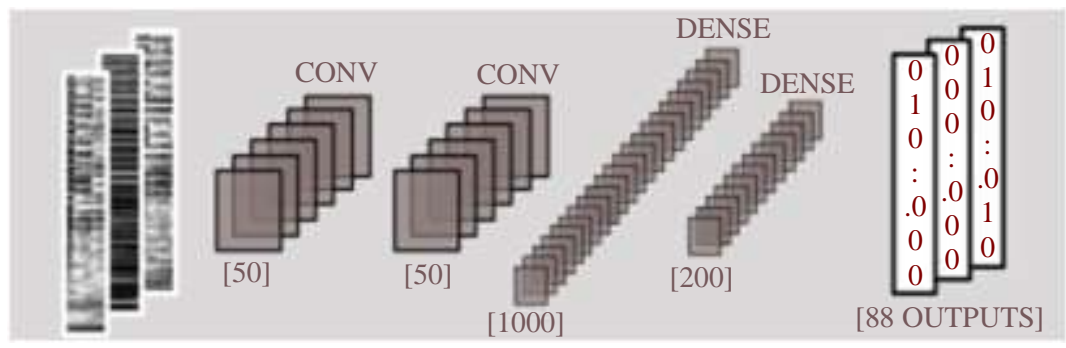

Fig. 4: CNN Architecture (Bereket and Shi, 2017)

\begin{tabular}{|c|c|c|c|c|c|}
\hline 1 & 1 & 1 & 1 & 1 & 1 \\
\hline 2 & 2 & 2 & 2 & 2 & 2 \\
\hline$\cdot$. &.. &.. &.. &.. & $\cdots$ \\
\hline 251 & 251 & 251 & 251 & 251 & 251 \\
\hline 252 & 252 & 252 & 252 & 252 & 252 \\
\hline
\end{tabular}

\begin{tabular}{|c|c|c|c|c|c|c|c|c|c|c|c|}
\hline 0 & 0 & 0 & 1 & 1 & 1 & 1 & 1 & 1 & 0 & 0 & 0 \\
\hline 0 & 0 & 0 & 2 & 2 & 2 & 2 & 2 & 2 & 0 & 0 & 0 \\
\hline 0 & 0 & 0 &.. &.. &.. &.. &.. &.. & 0 & 0 & 0 \\
\hline 0 & 0 & 0 & 251 & 251 & 251 & 251 & 251 & 251 & 0 & 0 & 0 \\
\hline 0 & 0 & 0 & 252 & 252 & 252 & 252 & 252 & 252 & 0 & 0 & 0 \\
\hline
\end{tabular}

Fig. 5: Padding in the CNN model

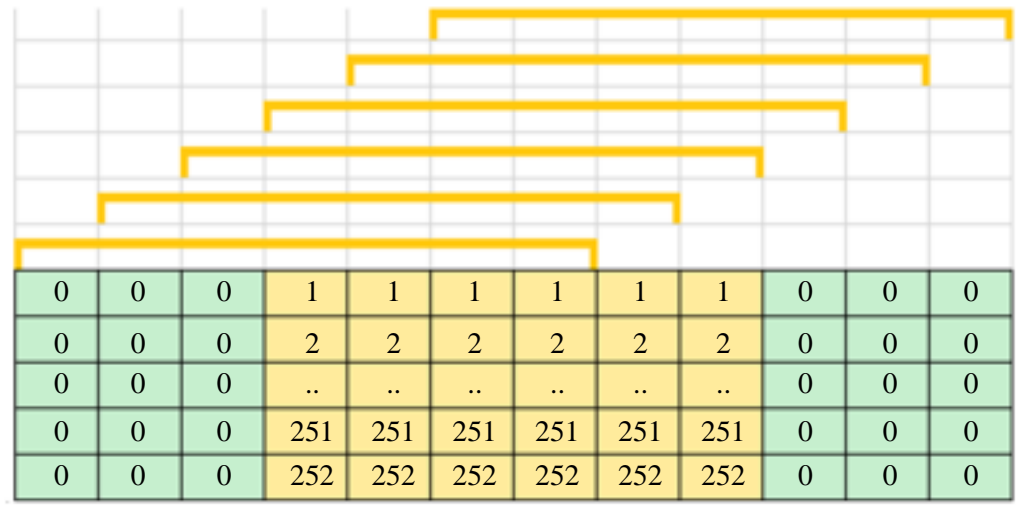

Fig. 6: Extracting windows visualization

In the case of CQT, zeros are added to the left and right side of the CQT as shown in Fig. 5. This is done to predict what is in the middle. The amount of padding will depend on the window size used.

Extracting windows will take several adjacent frames according to what window size is set to and put them together to a new array. For instance, when the window size is seven, then three frames on the left and three frames on the right are taken to form a single window as shown in Fig. 6 . This process is then repeated for each and every frame resulting in a three-dimensional array with the shape of 252 features times 7 frames and some number of windows depending on time which is annotated by -1 .

Once the preprocessing is done, the data file is opened and the data is loaded into memory. The data are then split into 5 groups that will then be split into train data which contains 4 groups and test data which contains 1 group. The models are then fitted 5 times all with different test data. At the end of each training session, scores such as precision, recall and F1 are recorded which then will be averaged when all 5 are done.

\section{Long Short-Term Memory}

Figure 7 shows that LSTM adds a new mechanism called memory block in the hidden layers of RNN. The memory blocks may decide which information to remember or which information to forget by using a special multiplicative unit called gates to control the flow of information inside the neural network. Each memory block contains an input gate (i) and output gate (o). The input gate controls the information that gets inside the hidden node and the output gate controls the output of a cell activation to the rest of the network. Then, the forget gate is added to decide which information to keep or to forget. This is what makes the LSTM special, because it scales the internal state of the cell before adding its calculations into the input stream. Thus, adaptively forgetting or resetting the memory of the cell network. 


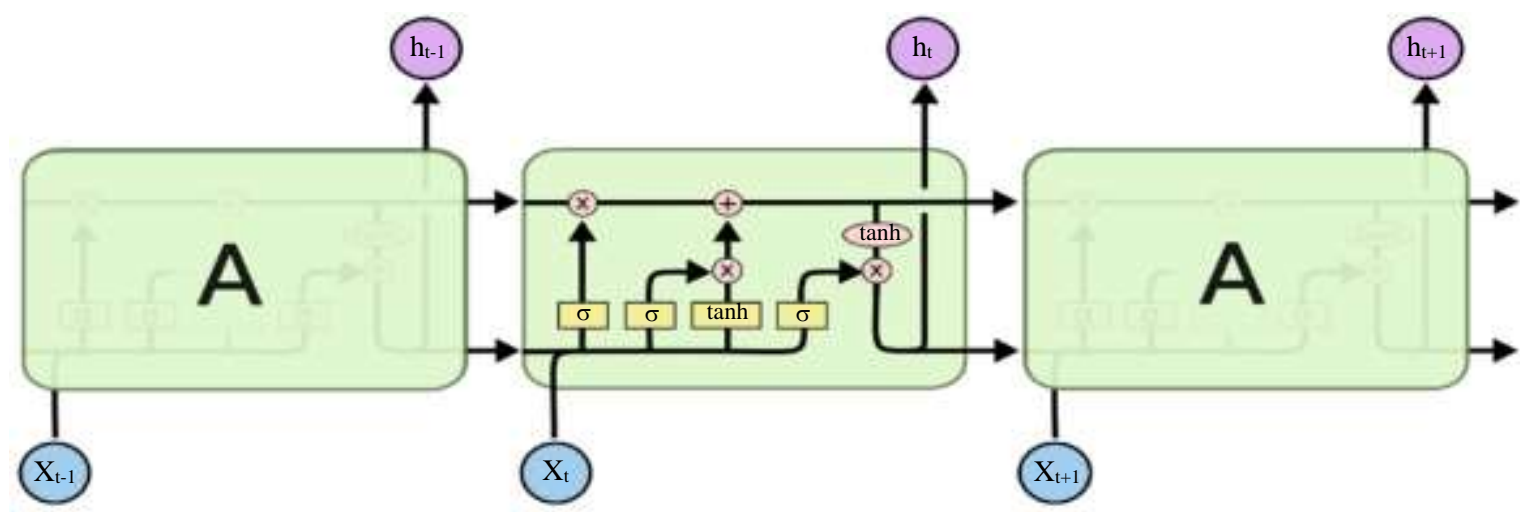

Fig. 7: An Individual Memory Cell of A LSTM Architecture (Morín, 2017)

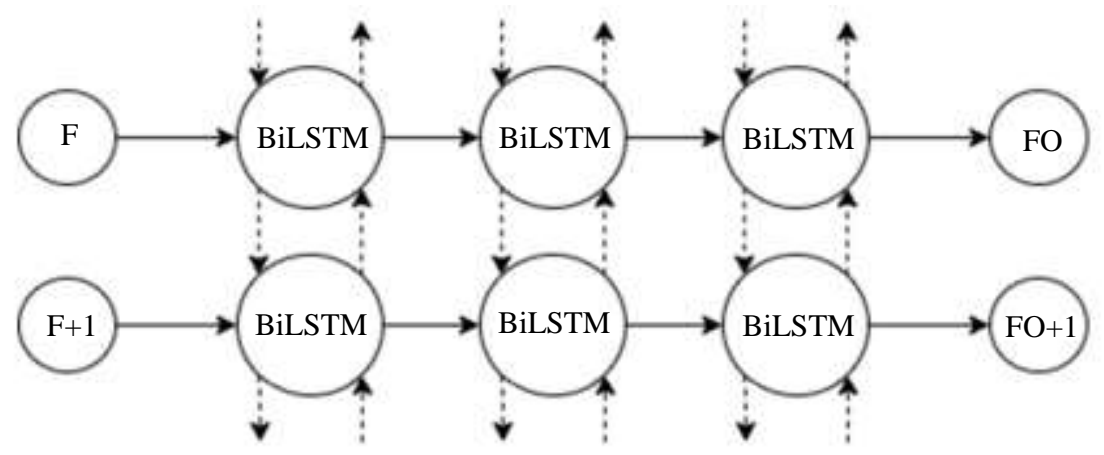

Fig. 8: BLSTM Architecture

In normalization, the maximum and minimum are recorded and then the values are capped to 0.0 to 1.0 . In the case of LSTM and DNN, an extra step is taken which is subtracting the mean from the data. This is done without modifying the shape of the CQT.

There are 3 hidden LSTM layers and each layer is as shown in the Fig. 8 (Morín, 2017), with each layer having 256 units. Using a batch size of 100 frames. The input matrix size is then modified from [frames, features] to [frames/100, 100, features] which makes the input size of the network [100, features]. Using hyperbolic tangent as the optimal activation function for LSTM. The output layer has 88 units using the sigmoid activation function. Mean Squared Error is used for the loss function with a dropout rate of 0.2 to avoid overfitting.

\section{Bidirectional Long Short-Term Memory}

Fs represent inputs whereas the FOs represent outputs. The dotted arrows symbolize the direction of the BiLSTM (forward or backward). The solid arrows symbolize the data going from one layer to the other layer.

The Bidirectional LSTM used has the exact same number of layers with its LSTM counterpart. There are 3 Bi-directional LSTM layers with the shape of $(100,256)$, followed by a dense or classification layer with the shape of $(100,88)$. This means that there are 3 LSTM layers going forward and 3 LSTM layers going backward with the shape of $(100,256)$ followed by a dense layer. At the very end of the BiLSTM layer, both outputs from the forward and backward LSTM layer are concatenated forming an array with 512 elements before being passed to the next layer. This means that not only will it know what is in the past but also what will come afterward, providing a better context.

\section{Results}

Table 1 shows the confusion matrix on class 7 of BiLSTM on a single music. The model predicted all of the true labels as false (false negatives). This happened as there is only a fraction of class 7 on the training data.

Table 2 shows the confusion matrix on class 54 of BiLSTM. Here, the model predicted most of the true labels as true some of them as false (false negatives) and predicted a fraction of false labels as true (false positives). The class scored 0.72 on F1-score. But note that this can be affected by the sample vector.

By looking at Table 3, it can be inferred that BiLSTM is superior when compared to the other architecture even though DNN has much higher epoch in most of the cases. 
Fernandes Saputra et al. / Journal of Computer Science 2021, 17 (3): 178.187 DOI: 10.3844/jessp.2021.178.187

Table 1: Confusion matrix on class 7 of BiLSTM

\begin{tabular}{lcc}
\hline & PRED true & PRED false \\
\hline True & 23079 & 521 \\
False & 0 & 0 \\
\hline
\end{tabular}

Table 2: Confusion matrix on class 54 of BiLSTM

\begin{tabular}{lcc}
\hline & PRED true & PRED false \\
\hline True & 19800 & 786 \\
False & 884 & 2130 \\
\hline
\end{tabular}

Table 3: Best evaluation of all the algorithms used 50 epoch and 20 early stop. (E - Epoch, A - Accuracy, P Precision, R - Recall, F1 - F-measure)

\begin{tabular}{lrllll}
\hline Name & E & A & P & R & F1 (\%) \\
\hline MLP & 13 & 96.78 & 69.85 & 67.08 & 67.86 \\
CNN & 22 & 96.68 & 51.30 & 14.70 & 22.85 \\
LSTM & 50 & 97.50 & 79.58 & 63.94 & 70.88 \\
Bi-LSTM & 93 & 97.82 & 83.09 & 68.06 & 74.80 \\
DNN & 152 & 97.63 & 80.05 & 66.66 & 72.74 \\
\hline
\end{tabular}

Table 4: Micro evaluation of CNN 50 epoch and 20 early stop

\begin{tabular}{llll}
\hline Accuracy (\%) & Precision (\%) & Recall (\%) & F1 (\%) \\
\hline 33.38 & 72.96 & 37.38 & 49.44 \\
\hline
\end{tabular}

When seeing MLP which scores around $67 \%$ in the table, it is quite surprising as the architecture used is naive and does not include any form of temporal context of the music as input. However, from both tables shown above it could be inferred that the MLP architecture is showing a massive indication of overfitting with every increase of epoch there is a decrease in the F1 score.

The CNN achieved underwhelming results 22.85 and $14.24 \%$ in micro and macro calculations which is below the naive MLP approach, when at least a score of $50 \%$ in the macro calculation was expected according to the proposed paper. Therefore, the authors tried out a lot of new hyper-parameter tuning and other cases solely for CNN architecture ranging from increasing the length of music (which easily triples the dataset samples), changing optimizer and changing learning rate but it only produces an insignificant or no improvement of the F1 score.

After all the tuning and changes that had been tested to $\mathrm{CNN}$, the figure is the only one to show significant improvement in CNN with $49.44 \%$ in the micro evaluation and $48.96 \%$ in the macro calculation shown in Table 4.

\section{Discussion}

High scores of LSTM and BiLSTM are kind of to be expected. The scores matched the scores proposed in its original paper with no problem at all. These two architectures have a context to learn from. When dealing with data that have a temporal dependency, context is important to make a prediction. For example, each word of a sentence such as this one depends on other words either before or after it to make sense. Now, a text or temporal prediction is similar to how a human makes a conclusion by reading the sentences back and forth. When LSTM is used, it can be considered as a fill-in-the-blank question with hints up to the blank, which depending on the question is possible but can be quite difficult even for humans. BiLSTM fixes this problem by providing hints not only up to the blank but also what comes after the blank forming a nearly complete fill-in-the-blank question (Ghaeini et al., 2018).

In theory as stated by (Li et al., 2017), LSTM was supposed to perform better than DNN due to the fact that LSTM takes temporal dependency into consideration, however the result shown in the Appendix Table 5 clearly shows that DNN F1 score is higher compared to LSTM. This case is similar to the result the previous research had found whereas the LSTM F1 score is outclassed compared to DNN. Similarly, LSTM has a smaller training loss and a larger validation loss which indicates an overfitting issue when compared to DNN.

Compared to research that had been done previously by (Kelz et al., 2016) and other similar research done by (Sigtia et al., 2016), the results that are shown in Appendix. Table 5 are definitely higher with a gap in the F1 score almost reaching to $7-17 \%$ when this research's BiLSTM is compared to their LSTM or DNN architecture. This research's LSTM and DNN also has a higher F1 score compared to their F1 score with almost $2-13 \%$ improvement.

Furthermore, the authors noticed that the highest F1 score that BiLSTM achieved (250 epoch case) doesn't improve that much from the previous case (100 epoch case), it also stopped around 89-93 epoch for both of the cases, this may indicate that BiLSTM has peak performance (the lowest validation loss as shown in the Fig. 9) that it could reach around that epoch and even if more epoch is added to the cases it may not help in increasing the evaluation score, worse to come, it might end up as an overfitted model similar to the MLP case:

weight $=n \cdot 9+1$

The above formula is to show the method used in improving the F1 score shown in Table 4 which is to use sample weight. For every sample, as the true label count increases, so does the weight which in this case uses the formula of the sum of true labels multiplied by 9 and added by one which gives us a weight of 1 for a completely silent frame, a weight of 10 for a note playing, 19 for two notes playing and so on. Which makes sense in a way since multiple notes playing together is rarer than a single note playing by itself. As shown in the Fig. 10. 


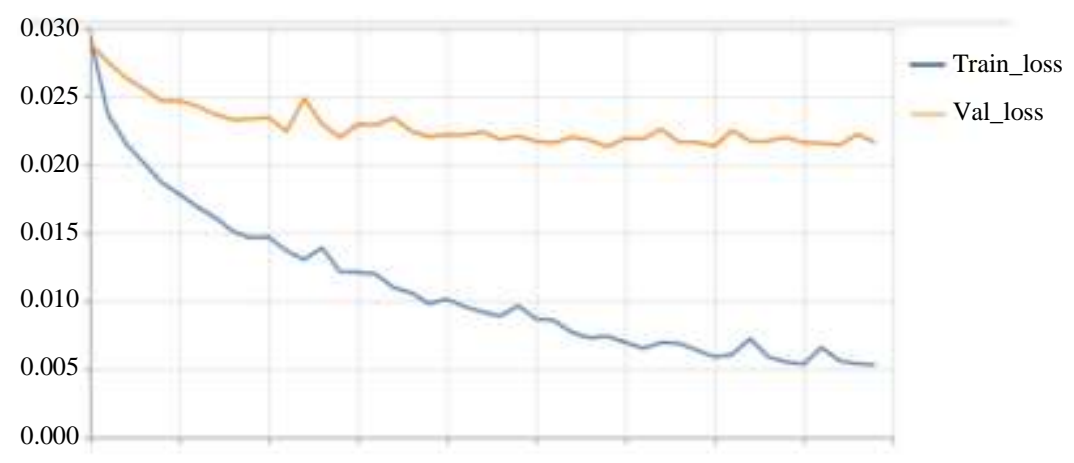

Fig. 9: BiLSTM 250 epoch data progression loss graph

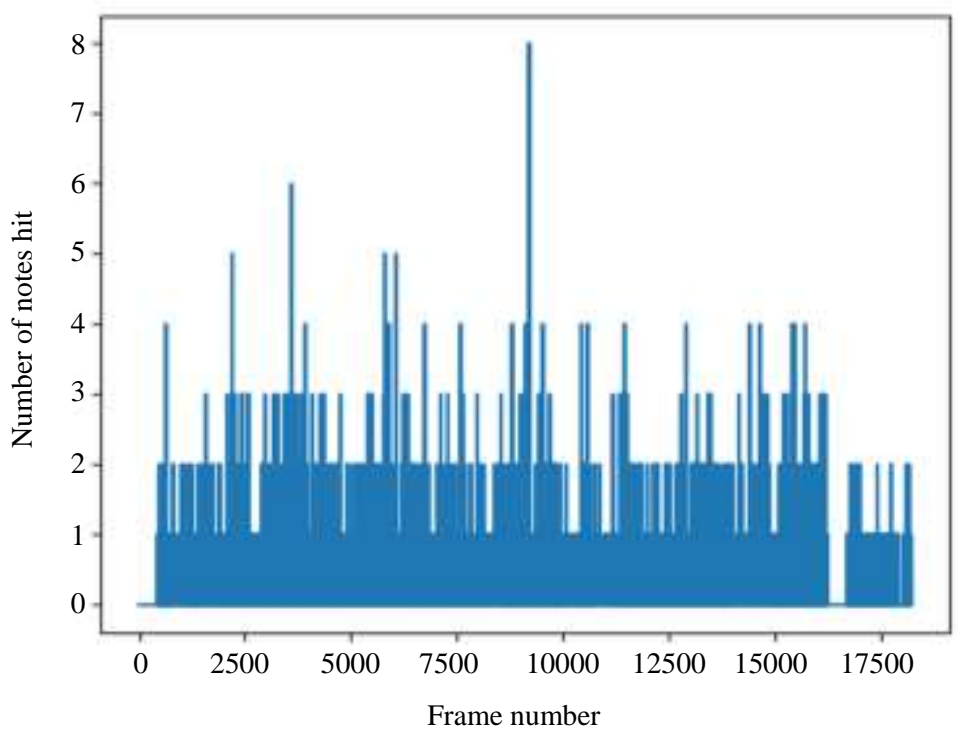

Fig. 10: Number of Notes Hit with Respect to Frame Number

\section{Conclusion and Future Works}

There are a few conclusions that could be extracted based on this research and the result of both objective evaluation and subjective evaluation of this research. First, out of all the architectures that were used and evaluated in this research, BiLSTM is able to give the best result for predicting key notes in a given song with F1 scoring as high as $74.80 \%$ in $80: 20$ configuration setting. Therefore, it could be concluded that BiLSTM is the best architecture suited for automatic music transcription. The last conclusion is that increasing the number of training epochs will only increase the evaluation metrics if the architecture has not reached the "limit" of improving with the given epochs. After passing a certain number, the said architecture does not improve as much or does not improve at all.

The suggestions that could be given to improve the method that is used in this research and the application is to improve the Convolutional Neural Network architecture into reaching a greater F1 score.
Then is to improve the F1 score of all the architectures by testing out different kinds of hyperparameter tuning and other kinds of post-processing methods such as RNN Neural Autoregressive Density Estimator (NADE). Another possible case is to test out different kinds of architecture combinations approach to automatic music transcription.

\section{Funding Information}

We would like to thank all friends and colleagues in School of Computer Science, Bina Nusantara University, for the supports. The author also would like to acknowledge Bina Nusantara University for supporting the whole research.

\section{Author's Contributions}

Fernandes Saputra: Contributed in writing the paper, coordinated teamwork, designed the whole research framework. 
Un Greffin Namyu: Coordinated the compilation of the dataset, participated in the implementation as well and contributed in manuscript writing.

Vincent: Played main role in the implementation of the research, elaborated a lot of resources to meet the requirements.

Derwin Suhartono: As well as corresponding author lead the team to contribute to the state of the art, managed all experiments finished in time, as well as ensured novelty in the manuscript.

Aryo Pradipta Gema: Directed all research works especially from machine learning perspective and also observed the whole manuscript before submission.

\section{Ethics}

This article is original and contains unpublished material. The corresponding author confirms that all of the other authors have read and approved the manuscript and no ethical issues involved.

\section{References}

Benetos, E., Dixon, S., Duan, Z., \& Ewert, S. (2018). Automatic music transcription: An overview. IEEE Signal Processing Magazine, 36(1), 20-30. https://doi.org/10.1109/MSP.2018.2869928

Bereket, M., \& Shi, K. (2017). An AI approach to automatic natural music transcription.

Blankertz, B. (2001). The constant Q transform. Retrieved from http://doc.ml.tuberlin.de/bbci/material/publications/Bla_constQ.pdf
Dua, M., Yadav, R., Mamgai, D., \& Brodiya, S. (2020). An Improved RNN-LSTM based Novel Approach for Sheet Music Generation. Procedia Computer Science, 171, 465-474. https://doi.org/10.1016/j.procs.2020.04.049

Emiya, V., Bertin, N., David, B., \& Badeau, R. (2010). MAPS-A piano database for multipitch estimation and automatic transcription of music.

Ghaeini, R., Hasan, S. A., Datla, V., Liu, J., Lee, K., Qadir, A., ... \& Farri, O. (2018). Dr-bilstm: Dependent reading bidirectional $1 \mathrm{stm}$ for natural language inference. arXiv preprint arXiv:1802.05577.

Hawthorne, C., Elsen, E., Song, J., Roberts, A., Simon, I., Raffel, C., ... \& Eck, D. (2017). Onsets and frames: Dual-objective piano transcription. arXiv preprint arXiv: 1710.11153.

Joshi, S., Kumari, A., Pai, P., Sangaonkar, S., \& D'Souza, M. (2017). Voice recognition system. Journal for Research, 3(01).

Kelz, R., Dorfer, M., Korzeniowski, F., Böck, S., Arzt, A., \& Widmer, G. (2016). On the potential of simple framewise approaches to piano transcription. arXiv preprint arXiv:1612.05153.

Li, L., Ni, I., \& Yang, L. (2017). Music Transcription Using Deep Learning.

Morín, D. G. (2017). Deep Neural Network for Piano Music Transcription. DT2119 Speech and Speaker Recognition course's project at KTH Royal Institute of Technology. Report of Deep Neural Networks for Piano Music Transcription.

Sigtia, S., Benetos, E., \& Dixon, S. (2016). An end-toend neural network for polyphonic piano music transcription. IEEE/ACM Transactions on Audio, Speech and Language Processing, 24(5), 927-939. https://doi.org/10.1109/TASLP.2016.2533858

\section{Appendix}

Table 5: Micro evaluation of all the architectures in every Scenario

\begin{tabular}{|c|c|c|c|c|c|c|c|}
\hline No & Architecture & Early stop & Epoch & Accuracy $(\%)$ & Precision $(\%)$ & Recall $(\%)$ & $\mathrm{F} 1(\%)$ \\
\hline 1 & MLP (20 Epoch) & 10 & 13 & $96.78 \pm 0.93$ & $69.85 \pm 9.42$ & $67.08 \pm 3.28$ & $67.86 \pm 3.96$ \\
\hline 2 & MLP (50 Epoch) & 15 & 19 & $96.76 \pm 0.79$ & $68.94 \pm 7.78$ & $67.01 \pm 3.10$ & $67.58 \pm 3.53$ \\
\hline 3 & MLP (100 Epoch) & 20 & 23 & $96.66 \pm 0.86$ & $67.98 \pm 8.75$ & $66.87 \pm 2.53$ & $66.95 \pm 3.66$ \\
\hline 4 & CNN (20 Epoch) & 10 & 12 & $96.67 \pm 0.12$ & $50.37 \pm 2.60$ & $12.72 \pm 1.13$ & $20.28 \pm 1.52$ \\
\hline 5 & CNN (50 Epoch) & 15 & 17 & $96.68 \pm 0.095$ & $51.52 \pm 3.16$ & $13.93 \pm 0.89$ & $21.93 \pm 1.36$ \\
\hline 6 & CNN (100 Epoch) & 20 & 22 & $96.68 \pm 0.089$ & $51.30 \pm 2.93$ & $14.70 \pm 0.82$ & $22.85 \pm 1.27$ \\
\hline 7 & LSTM (20 Epoch) & 10 & 20 & $97.33 \pm 0.2$ & $79.34 \pm 1.6$ & $59.23 \pm 3.7$ & $67.74 \pm 2.2$ \\
\hline 8 & LSTM (50 Epoch) & 15 & 50 & $97.50 \pm 0.2$ & $79.58 \pm 2.2$ & $63.94 \pm 2.7$ & $70.88 \pm 2.2$ \\
\hline 9 & LSTM (100 Epoch) & 20 & 78 & $97.46 \pm 0.2$ & $77.92 \pm 1.7$ & $64.88 \pm 2.7$ & $70.75 \pm 1.5$ \\
\hline 10 & LSTM (250 Epoch) & 20 & 73 & $97.42 \pm 0.2$ & $78.08 \pm 2.8$ & $63.54 \pm 2.9$ & $70.02 \pm 2.4$ \\
\hline 11 & Bidirectional LSTM (20 Epoch) & 10 & 20 & $97.45 \pm 0.2$ & $80.81 \pm 2.8$ & $60.70 \pm 3.2$ & $69.27 \pm 2.5$ \\
\hline 12 & Bidirectional LSTM (50 Epoch) & 15 & 49 & $97.72 \pm 0.2$ & $82.04 \pm 1.3$ & $66.50 \pm 3.4$ & $73.41 \pm 2.2$ \\
\hline 13 & Bidirectional LSTM (100 Epoch) & 20 & 89 & $97.80 \pm 0.2$ & $83.01 \pm 2.0$ & $67.49 \pm 2.9$ & $74.40 \pm 1.8$ \\
\hline 14 & Bidirectional LSTM (250 Epoch) & 20 & 93 & $97.82 \pm 0.2$ & $83.09 \pm 1.8$ & $68.06 \pm 2.7$ & $74.80 \pm 2.1$ \\
\hline 15 & DNN (20 Epoch) & 10 & 20 & $97.20 \pm 0.22$ & $78.40 \pm 2.26$ & $56.42 \pm 1.03$ & $65.60 \pm 1.16$ \\
\hline 16 & DNN (50 Epoch) & 15 & 50 & $97.48 \pm 0.24$ & $79.11 \pm 1.68$ & $63.69 \pm 2.04$ & $70.56 \pm 1.87$ \\
\hline 17 & DNN (100 Epoch) & 20 & 100 & $97.58 \pm 0.25$ & $78.81 \pm 1.76$ & $66.94 \pm 1.79$ & $72.39 \pm 1.80$ \\
\hline 18 & DNN (250 Epoch) & 20 & 152 & $97.63 \pm 0.26$ & $80.05 \pm 2.13$ & $66.66 \pm 2.02$ & $72.74 \pm 1.90$ \\
\hline
\end{tabular}

